\title{
Controlling Lateral Fano Interference Optical Force with Au-Ge2Sb2Te5 Hybrid Nanostructure
}

Cao, Tun; Bao, Jiaxin; Mao, Libang; Zhang, Tianhang; Novitsky, Andrey; Nieto-Vesperinas, Manuel; Qiu, Cheng-Wei

\section{Published in:}

ACS Photonics

Link to article, DOI:

10.1021/acsphotonics.6b00448

Publication date:

2016

Document Version

Peer reviewed version

Link back to DTU Orbit

Citation (APA):

Cao, T., Bao, J., Mao, L., Zhang, T., Novitsky, A., Nieto-Vesperinas, M., \& Qiu, C-W. (2016). Controlling Lateral Fano Interference Optical Force with Au-Ge2Sb2Te5 Hybrid Nanostructure. ACS Photonics, 3(10), 1934-1942. https://doi.org/10.1021/acsphotonics.6b00448

\section{General rights}

Copyright and moral rights for the publications made accessible in the public portal are retained by the authors and/or other copyright owners and it is a condition of accessing publications that users recognise and abide by the legal requirements associated with these rights.

- Users may download and print one copy of any publication from the public portal for the purpose of private study or research.

- You may not further distribute the material or use it for any profit-making activity or commercial gain

- You may freely distribute the URL identifying the publication in the public portal 


\title{
Controlling Lateral Fano Interference Optical Force with Au- $\mathrm{Ge}_{2} \mathrm{Sb}_{2} \mathrm{Te}_{5}$ Hybrid Nanostructure
}

\author{
Tun Cao, ${ }^{* \dagger}{ }^{\dagger}$ Jiaxin Bao, ${ }^{\dagger}$ Libang Mao, ${ }^{\dagger}$ Tianhang Zhang, ${ }^{\ddagger}$ Andrey Novitsky, ${ }^{\S, \perp}$ Manuel Nieto-Vesperinas, $"$ \\ and Cheng-Wei Qiu*,
}

\begin{abstract}
†Department of Biomedical Engineering, Dalian University of Technology, Dalian 116024, China
${ }^{\ddagger}$ Department of Electrical and Computer Engineering, National University of Singapore, 4 Engineering Drive 3, Singapore 117576, Republic of Singapore

${ }^{\S}$ Department of Theoretical Physics and Astrophysics, Belarusian State University, Nezavisimosti Avenue 4, 220030 Minsk, Belarus ${ }^{\perp}$ DTU Fotonik, Technical University of Denmark, Oersteds Plads 343, DK-2800 Kongens Lyngby, Denmark

"Instituto de Ciencia de Materiales de Madrid, Consejo Superior de Investigaciones Científicas, Campus de Cantoblanco, 28049 Madrid, Spain
\end{abstract}

\section{Supporting Information}

ABSTRACT: We numerically demonstrate that a pronounced dipolequadrupole (DQ) Fano resonance (FR) induced lateral force can be exerted on a dielectric particle $80 \mathrm{~nm}$ in radius $\left(R_{\text {sphere }}=80 \mathrm{~nm}\right)$ that is placed $5 \mathrm{~nm}$ above an asymmetric bow-tie nanoantenna array based on $\mathrm{Au} /$ $\mathrm{Ge}_{2} \mathrm{Sb}_{2} \mathrm{Te}_{5}$ dual layers. The DQ-FR-induced lateral force achieves a broad tuning range in the mid-infrared region by changing the states of the $\mathrm{Ge}_{2} \mathrm{Sb}_{2} \mathrm{Te}_{5}$ dielectric layer between amorphous and crystalline and in turn pushes the nanoparticle sideways in the opposite direction for a given wavelength. The mechanism of lateral force reversal is revealed through optical singularity in the Poynting vector. A thermal-electric simulation is adopted to investigate the temporal change of the $\mathrm{Ge}_{2} \mathrm{Sb}_{2} \mathrm{Te}_{5}$ film's temperature, which demonstrates the possibility of transiting the $\mathrm{Ge}_{2} \mathrm{Sb}_{2} \mathrm{Te}_{5}$ state by electrical heating. Our mechanism by tailoring the
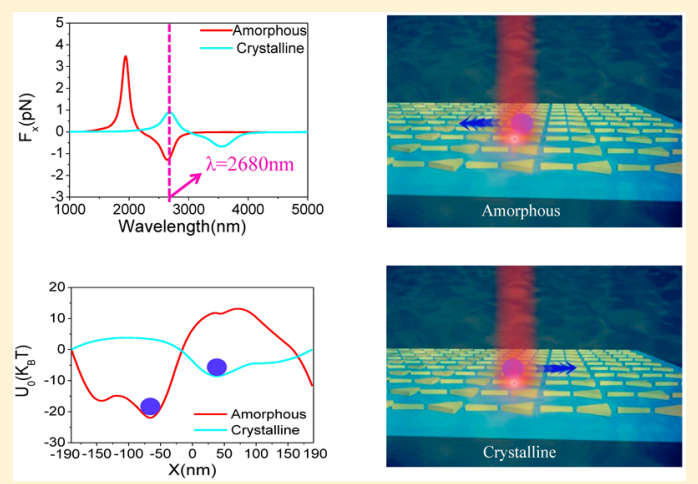
DQ-FR-induced lateral force presents clear advantages over the conventional nanoparticle manipulation techniques: it possesses a pronounced sideways force under a low incident light intensity of 10 $\mathrm{mW} / \mu \mathrm{m}^{2}$, a fast switching time of $2.6 \mu \mathrm{s}$, and a large tunable wavelength range. It results in a better freedom in flexible nanomechanical control and may provide a new means of biomedical sensing and nano-optical conveyor belts.

KEYWORDS: phase change material, Fano resonance, optical manipulation, surface plasmons, optical force

$\mathrm{O}$ ptomechanical manipulation of biomolecules using a focused laser beam has led to a host of applications in the biological and physical sciences. ${ }^{1,2}$ Even so, scaling this approach to nanoparticles is difficult since the limit of diffraction gives an upper bound in the gradient along light intensity and thus restrains the maximum gradient force that is proportional to the scale of the specimens for a given input power. $^{3}$ Since the optical trapping force increases with the local field gradient, nanostructures employed as auxiliary tools for optical trapping have been proposed to enhance the electromagnetic (EM) field and generate an evanescent field that gives a huge field gradient within a subwavelength area, e.g., slot waveguides, ${ }^{4}$ whispering-gallery-mode resonators, ${ }^{5}$ and photonic systems. ${ }^{6,7}$ Particularly, plasmonic optical tweezers are shown to be an influential approach to shrink the traditional optical tweezers to subwavelength scale. ${ }^{8-10}$ This scheme can break the diffraction limit since the optical trapping is based on localized surface plasmon resonance (LSPR) instead of propagating an EM wave. The LSPR mode confines the light within the diffraction limit, thus trapping the nanoparticle. Moreover, the LSPR mode's origin is the exponential decay of EM fields at the interface of the metal and dielectric, hence significantly enhancing the optical force caused by an intense field gradient. This leads to an achievement of stable optical trapping under a low incident light intensity. ${ }^{11-13}$ Therefore, plasmonic nanostructures such as nanorods, ${ }^{14}$ nanocavities, ${ }^{15}$ bow-tie nanoantennas, ${ }^{16}$ and coaxial apertures ${ }^{17}$ have been proposed to generate gradient optical force to trap molecules a few tens of nanometers in scale. The previous efforts commonly assumed that light could trap molecules using conservative gradient force induced by either bulky optical setups or compact nanodevices, whereas the position control of the nanoparticle in the plane perpendicular to the incident light using the conservative lateral force is rarely investigated.

Received: June 29, 2016 
Lately there is a growing attention to sensitive probing ${ }^{18}$ and enantiomer-selective separation of a racemic mixture through chiral forces. ${ }^{19-22}$ Even so, these strategies require circularly polarized light, which is more complicated to employ in comparison with linearly polarized light. Very recently, it has been shown that a plane wave with linear polarization can provide a nonconservative lateral force that drags nanosize helical specimens with different handedness along opposite directions. $^{23}$ However, the majority of studies of laterally nonconservative optical force aim at pushing chiral particles sideways, while the conservative lateral force on achiral particles and its application on optical manipulation are rarely investigated, such that the trace of a nonchiral particle changes at the molecular scale along the direction perpendicular to the incident light, which can behave as an optical conveyor belt to trap dielectric particles at different positions under controllable conditions. $^{24,25}$

Fano resonance (FR) can be understood as an interaction between a system with a broadened resonant line width and a system with a narrow resonance. ${ }^{26,27}$ FRs have been unveiled not only in a variety of plasmonic nanostructures ${ }^{28-36}$ but also in magnetodielectric ones. ${ }^{37-39}$ Despite its potential applications in nanoantennas, ${ }^{40,41}$ sensing, ${ }^{42-44}$ and optoelectronic devices, ${ }^{45,46}$ modern trends propose to study the dipolequadrupole (DQ) FR induced optical binding force (OBF) between plasmonic resonators. The direction of OBF is perpendicular to the incident light and can be reversed by tuning the gemometrical size and gap of the resonators. ${ }^{47,48}$ Inspired by these interesting phenomena, one important question arises: can we reverse the direction of the lateral force acting on nonchiral nanoparticles that is placed above the plasmonic nanostructure exhibiting DQ-FR.

Bow-tie nanoantennas (BNAs) have shown advantages over high numerical aperture (NA) lenses for optical trapping since they combine large field enhancements and subdiffraction field confinement properties within nanogaps through the LSPR. ${ }^{16,49}$ In this work, we numerically demonstrate an ultrafast reversible position controlling of nanoparticles using an actively tuned lateral DQ-FR force that is generated by an array of asymmetric BNAs embedded in a prototypical phase change material $\left(\mathrm{Ge}_{2} \mathrm{Sb}_{2} \mathrm{Te}_{5}\right)$ dielectric layer. The BNAs are designed to support a DQ-FR by introducing an asymmetry in the two tailored $\mathrm{Au}$ triangles to excite the nonradiative quadrupole mode. ${ }^{50}$ Unlike most prior plasmonic tweezers, here we will show that the DQ-FR in the asymmetric BNAs will induce a conservative lateral force that pushes the nanoparticles sideways rather than trap them. Moreover, the lateral optical force is reversibly altered, through a switchable tuning of the DQ-FR wavelength achieved by varying the state of $\mathrm{Ge}_{2} \mathrm{Sb}_{2} \mathrm{Te}_{5}$ between amorphous and crystalline. ${ }^{51}$ Consequently, it can change the sign of DQ-FR-induced transverse force at a fixed wavelength, hence moving the particle in the opposite direction. This advantage leads to a reversible manipulation of nanoparticles at linearly polarized plane wave incidence.

In this work, we show the DQ-FR-induced transverse force is related with the optical singularity in the Poynting vector (S). We explore the origin of transverse force as a result of a distinctive energy flow. It has been presented that the conservative lateral force is reversed via the manipulation of the distribution of singular points, which leads to a displacement of the hot spot around the nanoparticle, and the variation of the singular point distribution is obtained by a switchable state change of $\mathrm{Ge}_{2} \mathrm{Sb}_{2} \mathrm{Te}_{5}{ }^{51}$ Just as crucially, a thermoelectric simulation is performed to investigate the temporal change of the temperature of the $\mathrm{Ge}_{2} \mathrm{Sb}_{2} \mathrm{Te}_{5}$ dielectric layer. It illustrates that the amorphous $\mathrm{Ge}_{2} \mathrm{Sb}_{2} \mathrm{Te}_{5}$ layer's temperature can be increased from $293 \mathrm{~K}$ to $433 \mathrm{~K}$ (state change point of $\left.\mathrm{Ge}_{2} \mathrm{Sb}_{2} \mathrm{Te}_{5}\right)$ in $2.6 \mu \mathrm{s}$, under a biasing voltage of $20 \mathrm{~V}$. Thereby our proposed BNAs can rapidly alter the direction of DQ-FRinduced lateral force, which is very advantageous for biomedical applications where ultrafast manipulation of nano-objects is a key concern.

\section{RESULTS AND DISCUSSION}

The proposed BNA has two $15 \mathrm{~nm}$ thick $\mathrm{Au}$ isosceles triangles, the tip-to-base height of each triangle is $160 \mathrm{~nm}$, and the two triangles are placed tip-to-tip and spaced by a small distance of $10 \mathrm{~nm}$ to generate a strong near-field intensity with an intense field gradient. The BNAs are deposited on a $15 \mathrm{~nm}$ thick $\mathrm{Ge}_{2} \mathrm{Sb}_{2} \mathrm{Te}_{5}$ dielectric layer. Herein, we model two sets of BNAs starting from a symmetric resonator with two exactly the same isosceles triangles, where the tip angle of each triangle is $\theta_{1}=5^{\circ}$ (Figure 1a), and then increase the tip angle of the right
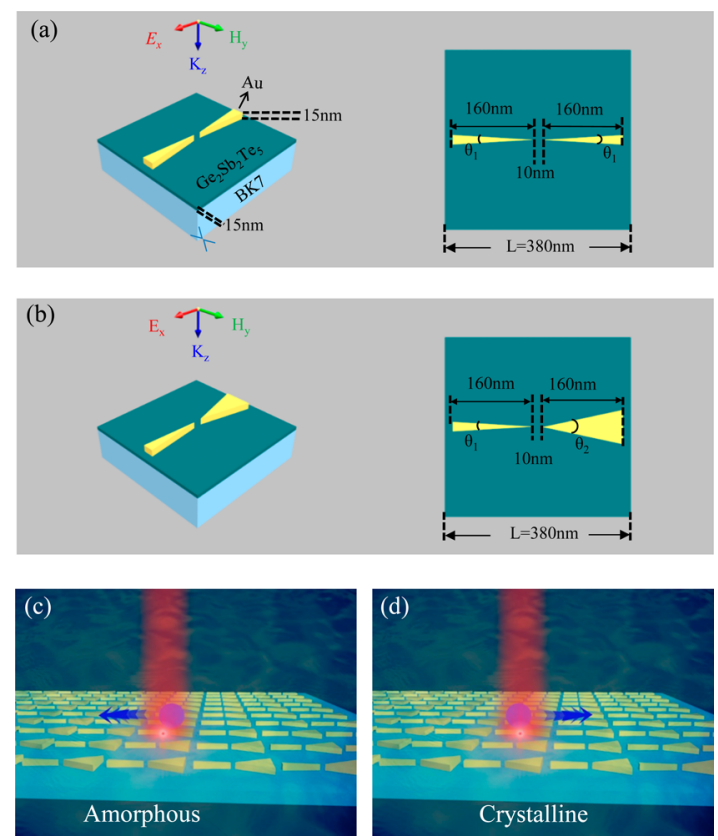

Figure 1. Schematic of a three-dimensional view of (a) symmetric BNAs and (b) asymmetric BNAs composed of Au BNAs embedded in the $\mathrm{Ge}_{2} \mathrm{Sb}_{2} \mathrm{Te}_{5}$ dielectric layer sitting on a BK7 glass substrate. A linearly polarized light from the top illuminates the structures. Right columns in (a) and (b) are the top view of the meta-atom. Illustration of the action of a dielectric sphere $\left(n_{\text {sphere }}=2, R_{\text {sphere }}=80 \mathrm{~nm}\right)$ in an asymmetric BNA optical system for the (c) amorphous state and (d) crystalline state. Blue arrows indicate the directions of the spheres' motions.

isosceles triangle to $\theta_{2}=25^{\circ}$ to break the symmetry (Figure 1b). For both structures, the lattice constant of the BNAs is $L=$ $380 \mathrm{~nm}$ and the Au bow-tie antennas are periodically placed in the $x$ and $y$ directions. The BNAs embedded in the $\mathrm{Ge}_{2} \mathrm{Sb}_{2} \mathrm{Te}_{5}$ dielectric layer are considered to stand on a BK7 glass substrate. $\mathrm{Au}$ is used as the metal owing to its nonoxidation and low ohmic loss. The geometry of the asymmetric bow-tie antenna and the $\mathrm{Ge}_{2} \mathrm{Sb}_{2} \mathrm{Te}_{5}$ layers' thickness are selected to allow for exerting a pronounced DQ-FR-induced lateral force on a dielectric particle $\left(R_{\text {sphere }}=80 \mathrm{~nm}\right)$, placed $5 \mathrm{~nm}$ above the 
surface of the asymmetric BNAs under a low illumination intensity of $10 \mathrm{~mW} / \mu \mathrm{m}^{2}$ for both amorphous (Figure 1c) and crystalline (Figure 1d) states, respectively. To approach a real situation, the system is placed in an environment of water. The dielectric particle $\left(n_{\text {sphere }}=2\right)$ is used for mimicking biological particles, such as proteins and viruses. Noted that, here, the dielectric nanosphere is neither plasmonic nor magnetodielectric; in other words the FRs considered in the model originate purely from the plasmonic BNA. The structures are calculated via commercial software (Lumerical FDTD Solutions) based on the finite difference time domain (FDTD) method. The size of the uniform mesh is set at 3 $\mathrm{nm}$ along all Cartesian axes $(\Delta x=\Delta y=\Delta z=3 \mathrm{~nm})$ in order to minimize the numerical errors. Perfectly matched layer (PML) boundary conditions are used along the $z$ direction and periodic boundaries along the $x$ and $y$ directions. This model is used to achieve the EM field distribution around the targeting particle to calculate the optical force. A Drude model is employed for the dielectric permittivity of $\mathrm{Au}, \varepsilon_{\mathrm{m}}(\omega)=1-\frac{\omega_{\mathrm{p}}{ }^{2}}{\left[\omega\left(\omega+\mathrm{i} \omega_{\mathrm{c}}\right)\right]}$ where $\omega_{\mathrm{p}}=1.37 \times 10^{16} \mathrm{rad} / \mathrm{s}$ is the plasma frequency and $\omega_{c}=4.08 \times 10^{13} \mathrm{rad} / \mathrm{s}$ is the scattering frequency for Au. ${ }^{52}$ The structures are excited by a linearly polarized plane wave having a range of wavelengths from 1000 to $5000 \mathrm{~nm}$, propagating through the $z$ direction with the $E$-field polarized in the transverse axis ( $x$ axis) of the bow-tie antenna (Figure 1).

$\mathrm{Ge}_{2} \mathrm{Sb}_{2} \mathrm{Te}_{5}$ is a chalcogenide glass having a crystallization temperature of $\sim 433 \mathrm{~K}$ and a melting temperature of $\sim 873 \mathrm{~K}^{53}$ The amorphous $\mathrm{Ge}_{2} \mathrm{Sb}_{2} \mathrm{Te}_{5}$ dielectric interlayer will be crystallized by heating it above the crystallization temperature, but without obtaining the melting temperature. A femtosecond (fs) laser with a relatively high power may amorphize the $\mathrm{Ge}_{2} \mathrm{Sb}_{2} \mathrm{Te}_{5}$, involving the melting and rapid quenching of the $\mathrm{Ge}_{2} \mathrm{Sb}_{2} \mathrm{Te}_{5}$ back to its amorphous state. It is because the fs laser generates a very high density of electron-hole pairs in the $\mathrm{Ge}_{2} \mathrm{Sb}_{2} \mathrm{Te}_{5}$ that the $\mathrm{Ge}_{2} \mathrm{Sb}_{2} \mathrm{Te}_{5}$ subsequently thermalizes and melts. Fast cooling quenches the melt into the amorphous state. Thus, $\mathrm{Ge}_{2} \mathrm{Sb}_{2} \mathrm{Te}_{5}$ has a potential of realizing modulation functionality, as it can reversibly switch between crystalline and amorphous states in the infrared region. ${ }^{54-58}$ The dielectric constant of $\mathrm{Ge}_{2} \mathrm{Sb}_{2} \mathrm{Te}_{5}$ for the mid-infrared (M-IR) range is presented in Supplementary Section S1.

The force acting on a dielectric particle located in timeharmonic EM fields can be attained through a linear momentum conservation, in which the linear momentum is either mechanical or field momentum. The sum of these two momentums is preserved. When a particle is illuminated under a light, the momentum is transferred from the optical field to the mechanical and thus leads to an optical force exerted on the particle. Therefore, the optical force relates to the variation of mechanical momentum $(p)$ with time $(\mathrm{d} p / \mathrm{d} t)$. The EM-field momentum flux in the linear medium of permittivity $\varepsilon$ and permeability $\mu$ is shown by the time-averaged Maxwell stress tensor $\langle\overleftrightarrow{T}\rangle$.

$$
\langle\overleftrightarrow{T}\rangle=\frac{1}{2} \operatorname{Re}\left[\varepsilon E E^{*}+\mu H H^{*}-\frac{1}{2}\left(\varepsilon|E|^{2}+\mu|H|^{2}\right) \overleftrightarrow{I}\right]
$$

where $\langle\ldots\rangle$ represents $\frac{1}{T} \int_{0}^{T} \ldots \mathrm{d} t$ with $T=\frac{2 \pi}{\omega}, H H^{*}$ and $E E^{*}$ the outer product of the fields, and $\overleftrightarrow{I}$ the matrix of identity.
The total time-averaged FR induced force $\left(F_{\text {total }}\right)$ exerted on the sphere is calculated using the Maxwell stress tensor formalism and is given by

$$
F_{\text {total }}=\oint_{\text {s }}\langle\overleftrightarrow{T}\rangle \hat{n} d S
$$

where $\hat{n}$ is the unit vector normal to the surface and $s$ the integration calculated over a closed surface that surrounds the bead. Here, an FDTD model is built up to calculate the EM fields at the surface of a square box surrounding the dielectric bead.

Figure 2a presents reflectance for the structures of symmetric and asymmetric BNAs under normal incidence, where the state
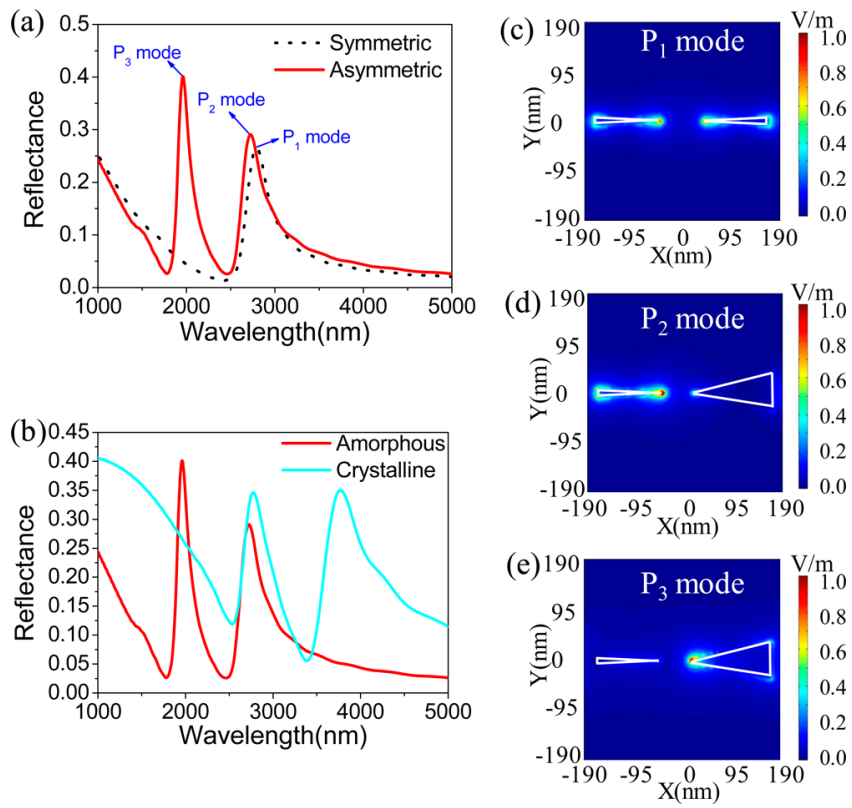

Figure 2. (a) Reflectance for both symmetric and asymmetric BNAs in an amorphous dielectric. (b) Reflectance comparison between the amorphous and crystalline asymmetric BNAs. Distribution of the $E$ field along the $\mathrm{Au} /$ water interface at (c) $\lambda=2788 \mathrm{~nm}$ for the amorphous symmetric BNAs and at (d) $\lambda=2727 \mathrm{~nm}$ and (e) $\lambda=1963$ $\mathrm{nm}$ for the amorphous asymmetric BNAs.

of the $\mathrm{Ge}_{2} \mathrm{Sb}_{2} \mathrm{Te}_{5}$ dielectric is amorphous. Here, we show that the symmetric BNAs exhibit a strong reflectance response with a resonant wavelength centered at $2788 \mathrm{~nm}$ (shown as a black dotted curve). By increasing the tip angle of the right triangle to $\theta_{2}=25^{\circ}$, the primary plasmon resonant mode for the symmetric BNAs breaks into two distinct resonant peaks at 1963 and $2727 \mathrm{~nm}$ by an asymmetric resonant dip at $2458 \mathrm{~nm}$ (shown as a red solid curve). In the case of the highly symmetric BNAs, only a bright dipolar mode that is strongly radiative in nature is excited. It is because the resonant modes in the two equal $\mathrm{Au}$ triangles overlap that they oscillate in phase and interfere constructively. In the asymmetric BNAs, a dark quadrupole mode is excited, which leads to a destructive interference between the bright dipolar mode of the small triangle $\left(\theta_{1}=5^{\circ}\right)$ and dark quadrupole mode of the large triangle $\left(\theta_{2}=25^{\circ}\right)$. The coherent coupling between the bright dipolar mode and dark quadrupole mode cancels the polarization of the bright dipolar mode, hence making a DQFR dip at $2458 \mathrm{~nm}$. We show that the enhanced FR can be achieved for the asymmetric BNAs since it possesses an ability to easily tune the dipolar and quadrupolar resonances by 
(a)

(b)

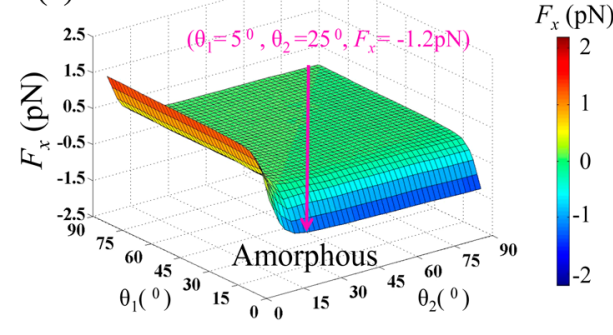

(b)

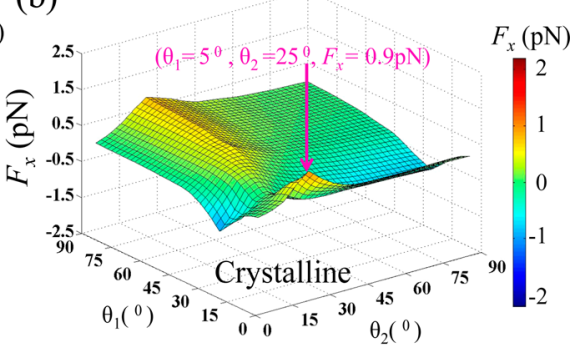

(c)

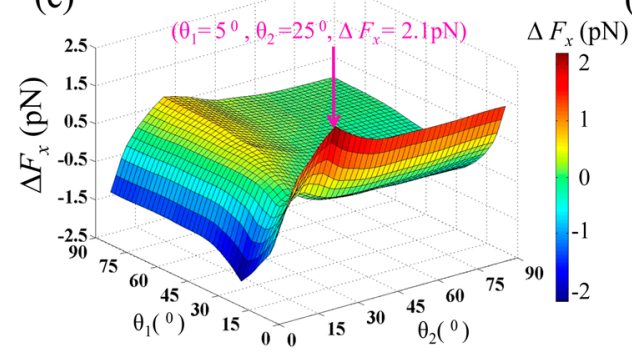

(d)

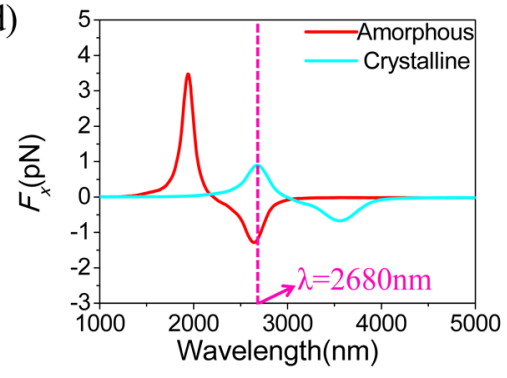

Figure 3. 2D diagram of lateral optical forces $\left(F_{x}\right)$ against $\theta_{1}$ and $\theta_{2}$ at a fixed wavelength of $2680 \mathrm{~nm}$, where the $F_{x}$ is exerted on the dielectric sphere $\left(R_{\text {sphere }}=80 \mathrm{~nm}\right)$ placed $5 \mathrm{~nm}$ above the asymmetric BNAs (a) with amorphous $\mathrm{Ge}_{2} \mathrm{Sb}_{2} \mathrm{Te}_{5}$ and (b) with crystalline $\mathrm{Ge}_{2} \mathrm{Sb}_{2} \mathrm{Te}_{5} ;(\mathrm{c}) 2 \mathrm{D}$ diagram of the difference of the $F_{x}\left(\Delta F_{x}\right)$ between the amorphous and crystalline asymmetric BNAs against $\theta_{1}$ and $\theta_{2}$ at a fixed wavelength of $2680 \mathrm{~nm}$; (d) comparison of the spectra of the $F_{x}$ between the amorphous and crystalline asymmetric BNAs with $\theta_{1}=5^{\circ}$ and $\theta_{2}=25^{\circ}$.

varying the geometry of the triangle, which determines the coupling strength between the neighboring plasmon modes in the bow-tie antenna. Figure $2 \mathrm{c}$ presents the intensity distribution of the total $E$-field $E=\sqrt{E_{x}^{2}+E_{y}^{2}+E_{z}^{2}}$ in the $x-y$ plane along the $\mathrm{Au} /$ water interface at $\lambda=2788 \mathrm{~nm}$ (denoted as the $\mathrm{P}_{1}$ mode) in the symmetric BNAs with amorphous $\mathrm{Ge}_{2} \mathrm{Sb}_{2} \mathrm{Te}_{5}$, where the color demonstrates the $E$ field intensities' magnitude. This shows that the distribution of the E-field intensity is symmetric and mainly concentrates within the tips of the bow-tie antenna. This indicates that the charges in the two equal bow-tie antennas oscillate in phase and interfere constructively (namely, the charges in the left and right tips of the gap are the same). Figure $2 \mathrm{~d}$ demonstrates the $E$-field intensity distribution at $\lambda=2727 \mathrm{~nm}$ (denoted as the $\mathrm{P}_{2}$ mode) for the asymmetric BNAs with amorphous $\mathrm{Ge}_{2} \mathrm{Sb}_{2} \mathrm{Te}_{5}$. The E-field intensity distribution at the $\mathrm{P}_{2}$ mode is more efficiently localized in the tip of the left, smaller triangle. Figure 2e shows the asymmetric BNAs also support the large E-field intensity in the tip of the right, larger triangle at the resonant wavelength of $\lambda=1963 \mathrm{~nm}$ (denoted as the $\mathrm{P}_{3}$ mode). As can be seen, the asymmetric BNAs provide the stronger electric resonances at the $P_{2}$ and $P_{3}$ modes compared to the $P_{1}$ mode in the symmetric BNAs. This is because, at the resonant mode of the asymmetric BNAs (i.e., DQ-FR), the charges in the left and right tips of the gap are opposite (namely, the charges in the two different bow-tie antennas oscillate out-of-phase and destructively interfere), which not only enhances the E-field intensity in the gap of the bow-tie nanoantenna but also resolves nanoscale mode volumes. Therefore, a laterally conservative optical force acting on a nanobead above asymmetric BNAs is larger than the symmetric BNAs, owing to the existence of the DQ-FR. Figure $2 b$ shows the evolution of the DQ-FR in reflectance spectra of the asymmetric BNAs as transiting the state of $\mathrm{Ge}_{2} \mathrm{Sb}_{2} \mathrm{Te}_{5}$ from amorphous to crystalline. With state variation of $\mathrm{Ge}_{2} \mathrm{Sb}_{2} \mathrm{Te}_{5}$, the DQ-FR dip at $\lambda=2458$ $\mathrm{nm}$ with an amorphous phase red-shifts to $3395 \mathrm{~nm}$ with a crystalline state. This is because a significant variation in $\operatorname{Re}\left(\varepsilon_{1}(\omega)\right.$ ) (a real part of dielectric permittivity $\varepsilon_{1}(\omega)$ ) of $\mathrm{Ge}_{2} \mathrm{Sb}_{2} \mathrm{Te}_{5}$ across the M-IR region is attained after altering the phase of thin film's structure from the amorphous to crystalline (Supplementary Section S1). This leads to a variation of the environmental dielectric constant of the BNAs and, in turn, tunes the spectra of the DQ-FR. Due to this large tuning range, the proposed phase-changed BNAs can be very useful in modulating the DQ-FR effect and sequentially provides a significant change of sign of the DQ-FR induced conservative lateral force at $2680 \mathrm{~nm}$, as shown in Figure 3d.

The total optical force $F_{\text {total }}$ can be resolved into two components: one is antiparallel or parallel to the propagation direction of incidence; the other is normal to the direction of the incidence, the so-called lateral optical force. In this work, we are focused on the lateral forces along the $x$ axis $\left(F_{x}\right)$, acting on the nonmagnetodielectric dielectric sphere $\left(R_{\text {sphere }}=80 \mathrm{~nm}\right)$ placed $5 \mathrm{~nm}$ above the asymmetric plasmonic BNAs with $\mathrm{Ge}_{2} \mathrm{Sb}_{2} \mathrm{Te}_{5}$. Here the main variables to be determined are the tip angles of $\theta_{1}$ and $\theta_{2}$ shown in Figure $1 \mathrm{~b}$. They are optimized for obtaining the maximum difference of $F_{x}$ between the amorphous and crystalline structures at a fixed wavelength of $\lambda$ $=2680 \mathrm{~nm}$. Figure $3 \mathrm{a}$ and $\mathrm{b}$ show $2 \mathrm{D}$ diagrams of $F_{x}$ against $\theta_{1}$ and $\theta_{2}$ for both the amorphous and crystalline structures, respectively, where the $F_{x}$ transits from -1.2 to $0.9 \mathrm{pN}$ with $\theta_{1}$ $=5^{\circ}$ and $\theta_{2}=25^{\circ}$ as the state of $\mathrm{Ge}_{2} \mathrm{Sb}_{2} \mathrm{Te}_{5}$ switches between amorphous and crystalline. The difference of $F_{x}\left(\Delta F_{x}\right)$ is defined as the subtraction of $F_{x}$ between the crystalline and amorphous states. In Figure $3 c$, it is shown the $\Delta F_{x}$ reaches the maximum value of $2.1 \mathrm{pN}$ at $\theta_{1}=5^{\circ}$ and $\theta_{2}=25^{\circ}$. To investigate how the $F_{x}$ is induced by the DQ-FR, Figure $3 \mathrm{~d}$ presents the comparison of the spectra of $F_{x}$ between the amorphous and crystalline phases. As presented by the red solid line in Figure $3 \mathrm{~d}$, for the amorphous asymmetric BNAs, the DQ-FR provides an asymmetric line shape in the spectra of the $F_{x}$. The $F_{x}$ is evident and undergoes a positive DQ-FR-induced lateral force $\left(F_{x}>0\right)$ across the blue region of the DQ-FR of 
$\lambda_{\text {Fano }}=2214 \mathrm{~nm}$ (the wavelength at which $F_{x}$ crosses the zero point, $F_{x}=0$ ), where $F_{x}$ repels the particle toward the $+x$ direction. For $\lambda>\lambda_{\text {Fano }}, F_{x}$ changes from positive to negative $\left(F_{x}<0\right)$ to drag the particle along the $-x$ direction. Namely, $F_{x}$ can be reversed when the wavelength of the incident plane wave increases from 1000 to $5000 \mathrm{~nm}$. Moreover, the $F_{x}$ exhibits a profoundly asymmetric resonance dip around $\lambda=$ $2680 \mathrm{~nm}$, indicating a strong negative force $\left(F_{x}=-1.2 \mathrm{pN}\right)$ under a low incident intensity of $10 \mathrm{~mW} / \mu \mathrm{m}^{2}$. To explore why $F_{x}$ at $\lambda=1938 \mathrm{~nm}$ is larger than that at $\lambda=2646 \mathrm{~nm}$ (shown in Figure $3 \mathrm{~d}$ ), we present the E-field intensity distribution in Supplementary Section S2.

Transiting the phase of $\mathrm{Ge}_{2} \mathrm{Sb}_{2} \mathrm{Te}_{5}$ from amorphous to crystalline (shown as a cyan solid line) spectrally tunes the DQFR in the asymmetric phase-changed BNAs, hence red-shifting the dip of $F_{x}$ from 2680 to $3550 \mathrm{~nm}$. It is noteworthy that around the wavelength of $2680 \mathrm{~nm}, F_{x}$ changes sharply in the opposite direction for the amorphous asymmetric BNAs $\left(F_{x}=\right.$ $-1.2 \mathrm{pN})$ and crystalline BNAs $\left(F_{x}=0.9 \mathrm{pN}\right)$. Therefore, reversibly switching the state of $\mathrm{Ge}_{2} \mathrm{Sb}_{2} \mathrm{Te}_{5}$ between amorphous and crystalline can change the signs of the $F_{x}$ exerted on the nanoparticle and, in turn, laterally manipulates the particle in the reverse direction. The higher order multipolar modes (the mode's order is higher than quadrupole) can also be excited in the right, large triangle $\left(\theta_{2}=25^{\circ}\right)$ to interfere with the dipolar mode in the left, small triangle $\left(\theta_{1}=5^{\circ}\right)$ and generate higher order FR. However, here these higher order modes' influence on the $F_{x}$ is neglected. The octupole resonance is comparatively weak due to the ultrasmall beads. Thereby, the optical forces induced by the higher order FR are not pronounced in the spectra of $F_{x}$ for the nanoparticles, and the quadrupole mode is enough for the convergence. To show the DQ-FR-induced $F_{x}$ agrees well with a Fano model, we approach the curve of $F_{x}$ acting on the dielectric sphere above both the amorphous and crystalline asymmetric BNAs, respectively, using the FR line shape in refs 26 and 27, as we demonstrate in Supplementary Section S3.

The lateral force induced by Fano interactions comes from the divergence of EM energy density, where the asymmetric BNAs are selected to concentrate the E-field to a very localized and intense hot spot, providing a large force density. ${ }^{59}$ Such a strong near-field hot spot leads to a pronounced enhancement in EM field strength and its gradient within this region. ${ }^{24,25}$ Therefore, the ability to control the location of hot spots by changing the state of $\mathrm{Ge}_{2} \mathrm{Sb}_{2} \mathrm{Te}_{5}$ between the crystalline and amorphous enables one to switch the direction of DQ-FRinduced lateral conservative force. To investigate the relation between the lateral force $F_{x}$ and near-field hot spot, the nearfield Poynting vector $(\mathbf{S})$ is demonstrated when the dielectric sphere is positioned $5 \mathrm{~nm}$ above the center of an asymmetric BNA for both crystalline and amorphous phases, respectively. $\mathbf{S}$ can provide an intuitive physical explanation of the matterwave interference, wherein $\mathbf{S}$ represents the flow of energy referring to the Poynting theorem. ${ }^{60}$ The first investigation of wavefront dislocations was performed by Nye and Berry. ${ }^{61}$ Lately, the optical singularities in $\mathbf{S}$ have been applied to study the underlying physics of distinctively optical micromanipulation. ${ }^{62}$ Crucially, FR and optical singularity are in fact closely interrelated. ${ }^{63}$ Therefore, the energy flux trajectories and optical singularity topology are enlightening to interpret the fundamental mechanism of QD-FR-caused force on the nanosphere. One can categorize the singular points of $\mathbf{S}$ as vortex, saddle, sink, focus, and node. ${ }^{64,65}$ The flows of energy vary with distributions of singular points and thus change the force acting on the nanoparticles. The streamlines of $\mathbf{S}$ and distributions of near-field singularities in the $x-z$ plane are illustrated in Figure 4, in order to expose the hot spot map. The
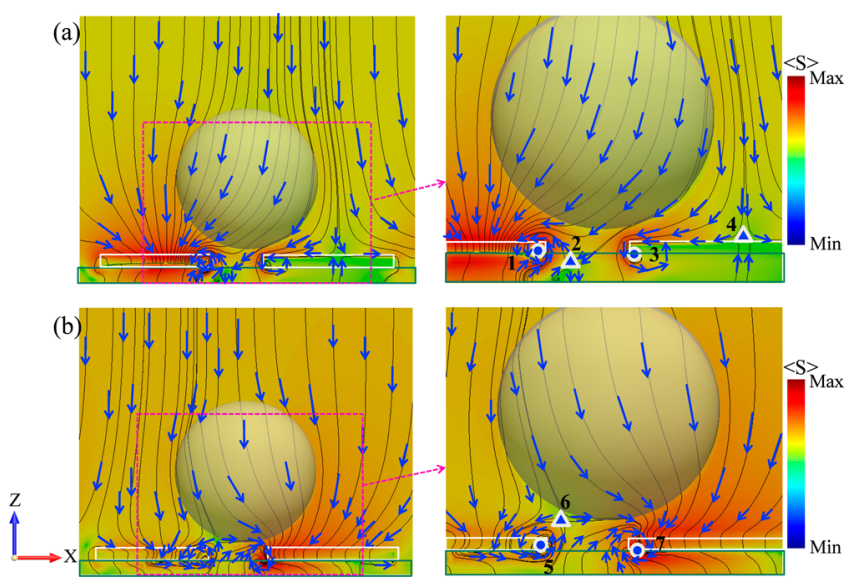

Figure 4. Field lines and intensity distributions of $\mathbf{S}$ for (a) a dielectric sphere placed $5 \mathrm{~nm}$ above the amorphous asymmetric BNAs and (b) a dielectric sphere placed $5 \mathrm{~nm}$ above the crystalline asymmetric BNAs. Right columns in (a) and (b) are the zoom-in pictures of the optical position control system based on the asymmetric phase change BNAs.

blue arrows and color bars represent the directions and logarithmic modulus of $\mathbf{S}$, accordingly. The streamline (black solid line) derives from a solution of $\frac{\mathrm{d} r}{\mathrm{~d} \varphi}=r \frac{\langle S\rangle_{\mathrm{r}}}{\langle S\rangle_{\varphi}}$, where $\langle S\rangle_{\varphi}$ and $\langle S\rangle_{\mathrm{r}}$ are the constituents of $\langle\mathbf{S}\rangle$ in polar coordinates. ${ }^{66}$ The $S$ in the amorphous asymmetric BNA at $\lambda=2680 \mathrm{~nm}$ is shown in Figure 4a. It shows that the hot spot is created at the tip of the left triangle of the BNAs. The field gradient near the hot spot results in a net $F_{x}$ toward the $-x$ direction $\left(F_{x}<0\right.$, red solid line in Figure $3 \mathrm{~d}$ ). In the vicinity of the tips of triangles, two pairs of saddle-vortex singularities appear. The saddle and vortex points unveil the significant variations in energy flow close to them. For example, the vortex points (marked with 1 and 3) "trap" the energy flux inside the whirlpool areas, and the saddle points (marked with 2 and 4) "seize" the energy flux from the surroundings. The LSPRs constructively interact with the incident light, creating a circle around vortex point 1 . Although vortex 3 and saddle 4 form a pair of saddle-vortex to direct the lightwave from the surroundings toward the bead's right side, the light energy is not pronouncedly improved. This is because the light passing through the right side of the sphere is guided to the left side through saddle 2 and encircles vortex 1. Consequently, the singular points may attract, guide, and congregate the light toward the tip of the right triangle and thus provides a hot spot.

For the crystalline BNA at $\lambda=2680 \mathrm{~nm}$, Figure $4 \mathrm{~b}$ shows the hot spot is shifted to the tip of the right triangle, thus altering the sign of $F_{x}\left(F_{x}>0\right.$, cyan solid line in Figure $\left.3 \mathrm{~d}\right)$. On the tip of the right triangle, a vortex point is created (marked with 7) to concentrate the E-field within the whirlpool region. Meanwhile a vortex-saddle pair (marked with 5 and 6) are excited on the tip of the left triangle. However, the lightwave travels across saddle point 6 , forms loops at vortex point 5, returns to saddle point 6 , and propagates to vortex point 7 . Therefore, the energy in the right side is stronger than that in the left side, resulting in a hot spot and thus a net lateral force along the $+x$ axis $\left(F_{x}>0\right)$. 
(a)
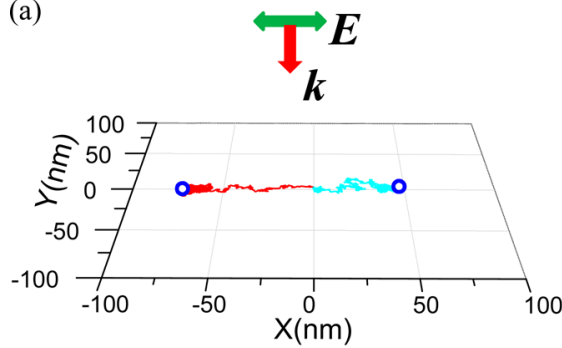

(b)

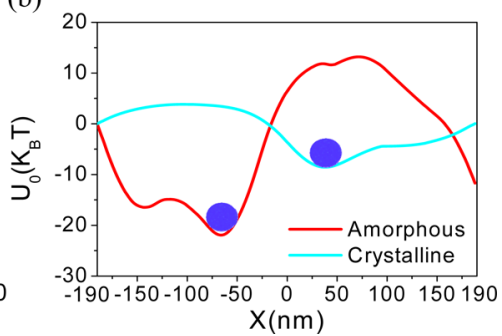

Figure 5. (a) Observation of the stability of a dielectric nanoparticle under lateral optical force. The red and cyan solid lines present the $0.2 \mathrm{~ms}$ trajectories of the dielectric spheres of $R_{\text {sphere }}=80 \mathrm{~nm}$, placed $5 \mathrm{~nm}$ above the asymmetric BNAs for both amorphous and crystalline $\mathrm{Ge}_{2} \mathrm{Sb}_{2} \mathrm{Te}_{5}$, respectively. The structures are normally illuminated by a plane wave with a low intensity of $10 \mathrm{~mW} / \mu \mathrm{m}^{2}$. The ends of the trajectories are indicated by small blue circles. The sphere is initially placed in the center of the plane $5 \mathrm{~nm}$ above the surface of the BNAs $(z=5 \mathrm{~nm}$ plane). (b) Optical potential $U_{0}$ as a function of the particle's positions along the $x$ axis with an excitation wavelength of $\lambda=2680 \mathrm{~nm}$, where the purple dots indicate the trapping positions of the particles, the $U_{0}$ distribution in the amorphous structure is indicated by the red solid line, and the crystalline structure is indicated by the cyan solid line. The environment is water $(T=300 \mathrm{~K})$.
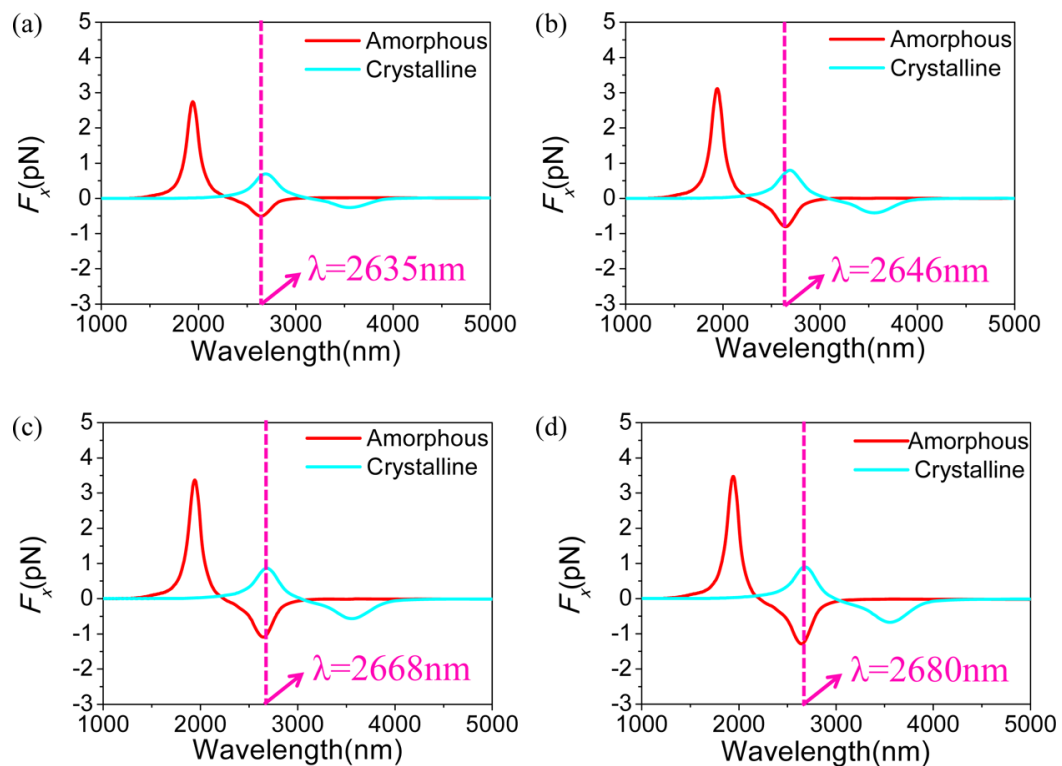

Figure 6. Comparison of the $F_{x}$ curve between the amorphous and crystalline asymmetric BNAs with $\theta_{1}=5^{\circ}$ and $\theta_{2}=25^{\circ}$, where $F_{x}$ is exerted on the dielectric spheres with different radius $\left(R_{\text {sphere }}\right)$ placed $5 \mathrm{~nm}$ above the asymmetric BNAs: $(\mathrm{a}) R_{\text {sphere }}=50 \mathrm{~nm},(\mathrm{~b}) R_{\text {sphere }}=60 \mathrm{~nm},(\mathrm{c}) R_{\text {sphere }}=70$ $\mathrm{nm},(\mathrm{d}) R_{\text {sphere }}=80 \mathrm{~nm}$.

Considering the random Brownian motion of nanoparticles in water, the stability of a nanoparticle must be examined. ${ }^{67,68}$ The dielectric particle's motion is modeled by the Langevin equation $^{24}$

$$
\begin{aligned}
& \frac{\mathrm{d}^{2} x(t)}{\mathrm{d} t^{2}}=-\frac{\beta}{m} \frac{\mathrm{d} x(t)}{\mathrm{d} t}+\frac{\zeta}{m} W_{x}(t)+\frac{F_{x}(x, y)}{m} \\
& \frac{\mathrm{d}^{2} y(t)}{\mathrm{d} t^{2}}=-\frac{\beta}{m} \frac{\mathrm{d} y(t)}{\mathrm{d} t}+\frac{\zeta}{m} W_{y}(t)+\frac{F_{y}(x, y)}{m}
\end{aligned}
$$

where $x(t)$ and $y(t)$ are the particle's positions, $F_{x}(x, y)$ and $F_{y}(x, y)$ are the lateral forces, $W_{x}(t)$ and $W_{y}(t)$ are the stochastic noise terms that model random collisions from fluid molecules along both the $x$ direction and $y$ direction, respectively, $m$ is the sphere's mass, $\beta=6 \pi \eta\left(R_{\text {sphere }}\right)$ is the drag coefficient (from Stoke's law for a spherical particle), and $\eta=0.89 \mathrm{mpa} \cdot \mathrm{s}$ is the viscosity of water. The scaling constant for the stochastic noise term is given by $\zeta=\sqrt{2 \beta k_{\mathrm{B}} T}$ where $k_{\mathrm{B}}$ is Boltzmann's constant and $T=300 \mathrm{~K}$. The simulation algorithm was run for a total of 200000 time steps with a very short time step of $1 \mathrm{~ns}$. Here, in order to simplify the simulation, we did not consider the influence of optical force along the $z$ direction on the motion of the particle.

In Figure 5a, we dynamically simulated the stability of nanoparticles by observing time sequences of the movements of a dielectric sphere that is placed $5 \mathrm{~nm}$ above the asymmetric BNAs with both amorphous and crystalline $\mathrm{Ge}_{2} \mathrm{Sb}_{2} \mathrm{Te}_{5}$, respectively. The particle in two dimensions $(x-y$ plane $)$ is tracked with nanometer precision. The intensity of the beam is $10 \mathrm{~mW} / \mu \mathrm{m}^{2}$ with an excitation wavelength of $\lambda=2680 \mathrm{~nm}$. A $0.2 \mathrm{~ms}$ trajectory of the sphere above the asymmetric BNAs with amorphous $\mathrm{Ge}_{2} \mathrm{Sb}_{2} \mathrm{Te}_{5}$ is shown by the red solid line. Owing to the location of the hot spot around the left tip of the triangle (see Figure $4 \mathrm{a}$ ), a lateral force $\left(F_{x}\right)$ is generated along the $-x$ axis $\left(F_{x}<0\right.$; see Figure $\left.3 \mathrm{~d}\right)$ and thus causes a motion of the particle to the left (Supplementary Movie S1). In contrast, within illumination by the plane wave beam, the nanoparticle above the crystalline structure follows the opposite direction of motion (see cyan solid line). This is because the hot spot shifted to the tip of the right triangle (see Figure $4 b$ ), where the $F_{x}$ generates along the $+x$ axis $\left(F_{x}>0\right)$, hence pushing the 

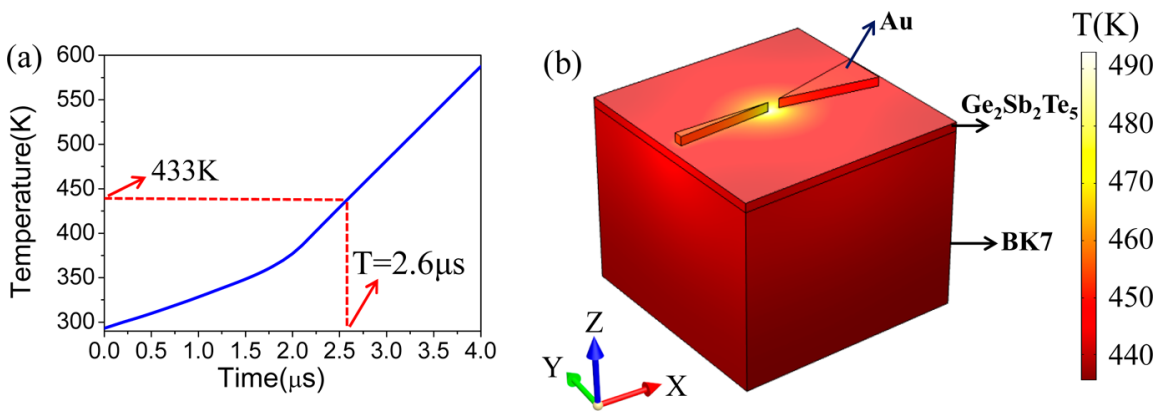

Figure 7. (a) Temperature of the $\mathrm{Ge}_{2} \mathrm{Sb}_{2} \mathrm{Te}_{5}$ dielectric layer in the asymmetric BNAs under $20 \mathrm{~V}$ bias voltage; (b) temperature distribution of the asymmetric BNAs at $2.6 \mu \mathrm{s}$, where the color image indicates the temperature values.

particle toward the right (Supplementary Movie S2). As can be seen in both Figure 5a and the supplementary movies, the nanoparticles thermally fluctuate only from -10 to $5 \mathrm{~nm}$ for the amorphous and from -8 to $14 \mathrm{~nm}$ for the crystalline state along the $y$ axis, which would not significantly affect the lateral position control of the spheres along the $x$ axis. The particles are stably trapped at the position of blue circles at the end of $0.2 \mathrm{~ms}$.

In Figure 5b, we show the transverse optical potential $U_{0}=$ $k_{\mathrm{B}} T$ created by the BNA for the nanoparticles $\left(R_{\text {sphere }}=80 \mathrm{~nm}\right)$ at different positions along the $x$ axis, where $T=300 \mathrm{~K}$ is the temperature of environmental water. The optical potential $U_{0}$ is the work of $F_{x}$ moving the particle from the central gap of the BNA to its arm along the $x$ axis. In the coordinate system, $F_{x}$ can provide a negative potential well $\left(U_{0}<0\right)$. For the asymmetric BNA with amorphous $\mathrm{Ge}_{2} \mathrm{Sb}_{2} \mathrm{Te}_{5}, U_{0}$ is negative $\left(U_{0}<0\right)$ along the $-x$ axis (see red solid line in Figure $5 b$ ), and a steep potential valley appears at $x=-66 \mathrm{~nm}$ corresponding to the potentials of DQ interference induced Fano lateral forces. Such a potential valley can drag the nanoparticle toward the left arm of the BNA and stably trap the nanoparticle around $x=$ $-66 \mathrm{~nm}$ (indicated by a purple dot). On transiting the state of $\mathrm{Ge}_{2} \mathrm{Sb}_{2} \mathrm{Te}_{5}$ from amorphous to crystalline, the potential valley of $U_{0}$ occurs at $x=+38 \mathrm{~nm}$, relating to the DQ-FR-induced $F_{x}$ (see cyan solid line in Figure $5 \mathrm{~b}$ ). Thus, the particle can be dragged to the right arm of the BNA and finally captured around $x=+38 \mathrm{~nm}$.

Furthermore, we investigate that such an asymmetric BNA can also produce a strong $F_{x}$ acting on the nanoparticle with a range of the radius from 50 to $80 \mathrm{~nm}$ owing to the DQ-FRs. As can be seen in Figure $6 a-d$, the Fano interaction between the dipolar and quadrupolar modes causes a prominent $F_{x}$ that can transit from the dip to peak at $\lambda=2635,2646,2668$, and 2680 $\mathrm{nm}$ for $R_{\text {sphere }}=50,60,70$, and $80 \mathrm{~nm}$, respectively, when the state of $\mathrm{Ge}_{2} \mathrm{Sb}_{2} \mathrm{Te}_{5}$ switches between the amorphous and crystalline. Although the absolute value of $F_{x}$ decreases with $R_{\text {sphere }} F_{x}$ still changes from -0.5 to $+0.6 \mathrm{pN}\left(\Delta F_{x}=1.1 \mathrm{pN}\right)$ for the smallest particle $\left(R_{\text {sphere }}=50 \mathrm{~nm}\right)$ during the phase transition, which is sufficient for the optical manipulation of the nanoparticles.

The most intriguing property of chalcogenide (i.e., $\mathrm{Ge}-\mathrm{Sb}-$ Te) plasmonics is its ultrabroad and fast tunability. One important concern for its practical applications is the response time of tunability. Recently, people have put much effort into shortening the time of state change in $\mathrm{Ge}-\mathrm{Sb}-\mathrm{Te}$ and experimentally demonstrated the prospect of acquiring a speed quicker than 100 ns. $^{69}$ These pave the way to ultrafast tuning of the $\mathrm{Ge}-\mathrm{Sb}-\mathrm{Te}$, which can provide a new scheme to obtain an immediate real-time position control of the specimen.
Electrical heating can be employed to achieve a reversible crystalline-amorphous phase change in $\mathrm{Ge}_{2} \mathrm{Sb}_{2} \mathrm{Te}_{5}$. Thus, it is important to study the switching behavior of asymmetric phasechange BNAs induced by electrical heating. Here, an alternative modeling approach has been developed on the basis of the finite element method (FEM) solver within COMSOL. First, the asymmetric BNAs based on amorphous $\mathrm{Ge}_{2} \mathrm{Sb}_{2} \mathrm{Te}_{5}$ are set up in COMSOL identical to that shown in Figure $1 \mathrm{~b}$.

Afterward, we carry out a simulation of heat transfer to study the temporal change of the $\mathrm{Ge}_{2} \mathrm{Sb}_{2} \mathrm{Te}_{5}$ layer's temperature under a biasing voltage of $20 \mathrm{~V}$. The material thermoelectric properties for $\mathrm{Ge}_{2} \mathrm{Sb}_{2} \mathrm{Te}_{5}, \mathrm{Au}$, and $\mathrm{BK} 7$ substrates used for the simulation are summarized in Supplementary Section S4. Figure $7 \mathrm{a}$ shows that the temperature in the $\mathrm{Ge}_{2} \mathrm{Sb}_{2} \mathrm{Te}_{5}$ dielectric layer in the amorphous state increases with the time of adding the biasing voltage of $20 \mathrm{~V}$. It goes beyond the amorphous to crystalline phase change temperature of $433 \mathrm{~K}$ after $2.6 \mu \mathrm{s}$. The 3D temperature distribution of the structure at $2.6 \mu \mathrm{s}$ is shown in Figure $7 \mathrm{~b}$. One can observe that the whole amorphous $\mathrm{Ge}_{2} \mathrm{Sb}_{2} \mathrm{Te}_{5}$ dielectric layer can exceed the transition temperature of $433 \mathrm{~K}$ after $2.6 \mu \mathrm{s}$, whereas the maximum temperature around $498 \mathrm{~K}$ is within the gap of the bow-tie antenna.

\section{CONCLUSION}

In conclusion, a method for ultrafast reversible manipulation of DQ-FR-induced lateral force created by an asymmetric BNA based on $\mathrm{Au} / \mathrm{Ge}_{2} \mathrm{Sb}_{2} \mathrm{Te}_{5}$ dual layers has been illustrated. We have presented that, by introducing a broken symmetry into the BNA, the Fano interaction between dipole and quadrupole modes results in a sideways force exerted on the dielectric nanoparticle. Such a Fano interference conservative force may laterally drag the particle in the opposite direction upon switching the state of $\mathrm{Ge}_{2} \mathrm{Sb}_{2} \mathrm{Te}_{5}$ between amorphous and crystalline. A model of heat transfer is performed to determine the change of transient temperature in the asymmetric BNAs through an electric-thermal process. Our numerical simulation envisages that a short phase change time of $2.6 \mu \mathrm{s}$ can be obtained with a low pumping intensity of $10 \mathrm{~mW} / \mu \mathrm{m}^{2}$, leading to an ultrafast position control of the nanobeads. Flexible manipulation of the magnitude and direction of the $F_{x}$ (lateral force) paves the way for observing collective phenomena of nano-objects and may be harnessed to afford a new twist of selfadaptive and active nano-optomechanical devices based on chalcogenide glass. 


\section{ASSOCIATED CONTENT}

\section{S Supporting Information}

The Supporting Information is available free of charge on the ACS Publications website at DOI: 10.1021/acsphotonics.6b00448.

Further details on the Fano model fit of $F_{x}$ and material thermoelectric properties (PDF)

Supporting movie 1 (AVI)

Supporting movie 2 (AVI)

\section{AUTHOR INFORMATION}

\section{Corresponding Authors}

*E-mail (T. Cao): caotun1806@dlut.edu.cn.

*E-mail (C.-W. Qiu): eleqc@nus.edu.sg.

\section{Notes}

The authors declare no competing financial interest.

\section{ACKNOWLEDGMENTS}

This work was supported by National Natural Science Foundation of China (Grant Nos. 61172059, 51302026), International Science \& Technology Cooperation Program of China (Grant No. 2015DFG12630), and Program for Liaoning Excellent Talents in University (Grant No. LJQ2015021).

\section{REFERENCES}

(1) Grier, D. G. A Revolution in Optical Manipulation. Nature 2003, 424, 810-816.

(2) Gieseler, J.; Deutsch, B.; Quidant, R.; Novotny, L. Subkelvin Parametric Feedback Cooling of a Laser-trapped Nanoparticle. Phys. Rev. Lett. 2012, 109, 103603.

(3) Shvedov, V. G.; Rode, A. V.; Izdebskaya, Y. V.; Desyatnikov, A. S.; Krolikowski, W.; Kivshar, Y. S. Giant Optical Manipulation. Phys. Rev. Lett. 2010, 105, 118103-118104.

(4) Yang, A. H. J.; Moore, S. D.; Schmidt, B. S.; Klug, M.; Lipson, M.; Erickson, D. Optical Manipulation of Nanoparticles and Biomolecules in Sub-Wavelength Slot Waveguides. Nature 2009, 457, 71-75.

(5) Lin, S.; Schonbrun, E.; Crozier, K. Optical Manipulation with Planar Silicon Microring Resonators. Nano Lett. 2010, 10, 2408-2411.

(6) Wang, Z.; Rakich, P. T. Response Theory of Optical Forces in Two-Port Photonics Systems: A Simplified Framework for Examining Conservative and Non-Conservative Forces. Opt. Express 2011, 19, 22322-22336.

(7) Rakich, P. T.; Wang, Z.; Davids, P. Scaling of Optical Forces in Dielectric Waveguides: Rigorous Connection between Radiation Pressure and Dispersion. Opt. Lett. 2011, 36, 217-219.

(8) Juan, M. L.; Righini, M.; Quidant, R. Plasmon Nano-Optical Tweezers. Nat. Photonics 2011, 5, 349-356.

(9) Quidant, R. Plasmonic Tweezers-The Strength of Surface Plasmons; MRS Bull: Cambridge Univ Press, 2012.

(10) Berthelot, J.; Acimovic, S. S.; Juan, M. L.; Kreuzer, M. P.; Renger, J.; Quidant, R. Three-Dimensional Manipulation with Scanning Near-Field Optical Nanotweezers. Nat. Nanotechnol. 2014, 9, 295-299.

(11) Zhang, W.; Huang, L.; Santschi, C.; Martin, O. J. F. Trapping and Sensing $10 \mathrm{~nm}$ Metal Nanoparticles Using Plasmonic Dipole Antennas. Nano Lett. 2010, 10, 1006-1011.

(12) Yang, X.; Liu, Y.; Oulton, R. F.; Yin, X.; Zhang, X. Optical Forces in Hybrid Plasmonic Waveguides. Nano Lett. 2011, 11, 321328.

(13) Huidobro, P. A.; Ota, S.; Yang, X.; Yin, X.; Garcia-Vidal, F. J.; Zhang, X. Plasmonic Brownian Ratchet. Phys. Rev. B: Condens. Matter Mater. Phys. 2013, 88, 201401.

(14) Tanaka, Y.; Sasaki, K. Optical Trapping through the Localized Surface-plasmon Resonance of Engineered Gold Nanoblock Pairs. Opt. Express 2011, 19, 17462-17468.
(15) Chen, C.; Juan, M. L.; Li, Y.; Maes, G.; Borghs, G.; Drope, P. V.; Quidant, R. Enhanced Optical Trapping and Arrangement of Nanoobjects in a Plasmonic Nanocavity. Nano Lett. 2011, 12, 125132.

(16) Roxworthy, B. J.; Ko, K. D.; Kumar, A.; Fung, K. H.; Chow, E. K.; Liu, G. L.; Fang, N. X.; Toussaint, K. C., Jr Application of Plasmonic Bowtie Nanoantenna Arrays for Optical Trapping, Stacking, and Sorting. Nano Lett. 2012, 12, 796-801.

(17) Saleh, A. A. E.; Dionne, J. A. Toward Efficient Optical Trapping of Sub-10-nm Particles with Coaxial Plasmonic Apertures. Nano Lett. 2012, 12, 5581-5586.

(18) Bliokh, K. Y.; Kivshar, Y. S.; Nori, F. Magnetoelectric Effects in Local Light-Matter Interactions. Phys. Rev. Lett. 2014, 113, 033601.

(19) Tkachenko, G.; Brasselet, E. Spin Controlled Optical Radiation Pressure. Phys. Rev. Lett. 2013, 111, 033605.

(20) Tkachenko, G.; Brasselet, E. Optofluidic Sorting of Material Chirality by Chiral Light. Nat. Commun. 2014, 5, 3577.

(21) Tkachenko, G.; Brasselet, E. Helicity-dependent Three-dimensional Optical Trapping of Chiral Microparticles. Nat. Commun. 2014, 5, 4491.

(22) Alizadeh, M. H.; Reinhard, B. M. Plasmonically Enhanced Chiral Optical Fields and Forces in Achiral Split Ring Resonators. ACS Photonics 2015, 2, 361-368.

(23) Wang, S. B.; Chan, C. T. Lateral Optical Force on Chiral Particles Near a Surface. Nat. Commun. 2014, 5, 3307.

(24) Hansen, P.; Zheng, Y.; Ryan, J.; Hesselink, L. Nano-Optical Conveyor Belt, Part I: Theory. Nano Lett. 2014, 14, 2965.

(25) Zheng, Y.; Ryan, J.; Hansen, P.; Cheng, Y.; Lu, T.; Hesselink, L. Nano-optical Conveyor Belt, Part II: Demonstration of Handoff. Nano Lett. 2014, 14, 2971-2976.

(26) Fano, U. Effects of Configuration Interaction on Intensities and Phase Shifts. Phys. Rev. 1961, 124, 1866.

(27) Miroshnichenko, A. E.; Flach, S.; Kivshar, Y. S. Fano Resonances in Nanoscale Structures. Rev. Mod. Phys. 2010, 82, 2257.

(28) Shen, Y.; Rinnerbauer, V.; Wang, I.; Stelmakh, V.; Joannopoulos, J. D.; Soljacic, M. Structural Colors from Fano Resonances. ACS Photonics 2015, 2, 27-32.

(29) Zhang, Y.; Zhen, Y. R.; Neumann, O.; Day, J. K.; Nordlander, P.; Halas, N. J. Coherent Anti-Stokes Raman Scattering with Singlemolecule Sensitivity using a Plasmonic Fano Resonance. Nat. Commun. 2014, 5, 4424.

(30) Hopkins, B.; Filonov, D. S.; Miroshnichenko, A. E.; Monticone, F.; Alu, A.; Kivshar, Y. S. Interplay of Magnetic Responses in All Dielectric Oligomers to Realize Magnetic Fano Resonances. ACS Photonics 2015, 2, 724-729.

(31) Liu, N.; Mukherjee, S.; Bao, K.; Li, Y.; Brown, L. V.; Nordlander, P.; Halas, N. J. Manipulating Magnetic Plasmon Propagation in Metallic Nanocluster Networks. ACS Nano 2012, 6, 5482-5488.

(32) Luk'yanchuk, B.; Zheludev, N. I.; Maier, S. A.; Halas, N. J.; Nordlander, P.; Giessen, H.; Chong, C. T. The Fano Resonance in Plasmonic Nanostructures and Metamaterials. Nat. Mater. 2010, 9, 707-715.

(33) Srivastava, Y. K.; Manjappa, M.; Cong, L.; Cao, W.; Al-Naib, I.; Zhang, W.; Singh, R. Ultrahigh-Q Fano Resonances in Terahertz Metasurfaces: Strong Influence of Metallic Conductivity at Extremely Low Asymmetry. Adv. Opt. Mater. 2016, 4, 3.

(34) Dobmann, S.; Kriesch, A.; Ploss, D.; Peschel, U. Near-Field Analysis of Bright and Dark Modes on Plasmonic Metasurfaces Showing Extraordinary Suppressed Transmission. Adv. Opt. Mater. 2014, 2, 10.

(35) Chong, K. E.; Hopkins, B.; Staude, I.; Miroshnichenko, A. E.; Dominguez, J.; Decker, M.; Neshev, D. N.; Brener, I.; Kivshar, Y. S. Observation of Fano Resonances in All-Dielectric Nanoparticle Oligomers. Small 2014, 10, 1985-1990.

(36) Hopkins, B.; Poddubny, A. N.; Miroshnichenko, A. E.; Kivshar, Y. S. Circular Dichroism Induced by Fano Resonances in Planar Chiral Oligomers. Laser Photonics Rev. 2016, 10, 137-146. 
(37) Filonov, D. S.; Slobozhanyuk, A. P.; Krasnok, A. E.; Belov, P. A.; Nenasheva, E. A.; Hopkins, B.; Miroshnichenko, A. E.; Kivshar, Y. S. Appl. Phys. Lett. 2014, 104, 021104.

(38) Miroshnichenko, A. E.; Kivshar, Y. S. Fano Resonances in Alldielectric Oligomers. Nano Lett. 2012, 12, 6459-6463.

(39) Geffrin, J. M.; García-Cámara, B.; Gómez-Medina, R; Albella, P.; Froufe-Pérez, L. S.; Eyraud, C.; Litman, A.; Vaillon, R.; González, F.; Nieto-Vesperinas, M.; Sáenz, J. J.; Moreno, F. Magnetic and Electric Coherence in Forward-and Back-scattered Electromagnetic Waves by a Single Dielectric Subwavelength Sphere. Nat. Commun. 2012, 3, 1171.

(40) Liu, N.; Wen, F. F.; Zhao, Y.; Wang, Y.; Nordlander, P.; Halas, N. J.; Alu, A. Individual Nanoantennas Loaded with ThreeDimensional Optical Nanocircuit. Nano Lett. 2013, 13, 142-147.

(41) Li, Z. P.; Shegai, T.; Haran, G.; Xu, H. X. Multiple-Particle Nanoantennas for Enormous Enhancement and Polarization Control of Light Emission. ACS Nano 2009, 3, 637-642.

(42) King, N. S.; Liu, L.; Yang, X.; Cerjan, B.; Everitt, H. O.; Nordlander, P.; Halas, N. J. Fano Resonant Aluminum Nanoclusters for Plasmonic Colorimetric Sensing. ACS Nano 2015, 9, 1062810636.

(43) Hao, F.; Nordlander, P.; Sonnefraud, Y.; Van Dorpe, P.; Maier, S. A. Tunability of Subradiant Dipolar and Fano-Type Plasmon Resonances in Metallic Ring/Disk Cavities: Implications for Nanoscale Optical Sensing. ACS Nano 2009, 3, 643-652.

(44) Lütolf, F.; Casari, D.; Gallinet, B. Low-Cost and Large-Area Strain Sensors Based on Plasmonic Fano Resonances. Adv. Opt. Mater. 2016, 4, 5 .

(45) Liu, N.; Weiss, T.; Mesch, M.; Langguth, L.; Eigenthaler, U.; Hirscher, M.; Soennichsen, C.; Giessen, H. Planar Metamaterial Analogue of Electromagnetically Induced Transparency for Plasmonic Sensing. Nano Lett. 2010, 10, 1103-1107.

(46) Dabidian, N.; Kholmanov, I.; Khanikaev, A. B.; Tatar, K.; Trendafilov, S.; Mousavi, S. H.; Magnuson, C.; Ruoff, R. S.; Shvets, G. Electrical Switching of Infrared Light Using Graphene Integration with Plasmonic Fano Resonant Metasurfaces. ACS Photonics 2015, 2, 216227.

(47) Zhang, Q.; Xiao, J. J.; Zhang, X. M.; Yao, Y.; Liu, H. Reversal of Optical Binding Force by Fano Resonance in Plasmonic Nanorod Heterodimer. Opt. Express 2013, 21, 6601-6608.

(48) Zhang, J. F.; MacDonald, K. F.; Zheludev, N. I. Giant Optical Forces in Planar Dielectric Photonic Metamaterials. Opt. Lett. 2014, 39, 4883-4886.

(49) Pacheco-Peña, V.; Beruete, M.; Fernández-Domínguez, A. I.; Luo, Y.; Navarro-Cía, M. Description of Bow-Tie Nanoantennas Excited by Localized Emitters Using Conformal Transformation. ACS Photonics 2016, 3, 1223-1232.

(50) Fedotov, V. A.; Rose, M.; Prosvirnin, S. L.; Papasimakis, N.; Zheludev, N. I. Sharp Trapped-Mode Resonances in Planar Metamaterials with a Broken Structural Symmetry. Phys. Rev. Lett. 2007, 99, 147401.

(51) Shportko, K.; Kremers, S.; Woda, M.; Lencer, D.; Robertson, J.; Wuttig, M. Resonant Bonding in Crystalline Phase-change Materials. Nat. Mater. 2008, 7, 653-658.

(52) Zhang, S.; Fan, W.; Panoiu, N. C.; Malloy, K. J.; Osgood, R. M.; Brueck, S. R. Experimental Demonstration of Near-Infrared NegativeIndex Metamaterials. Phys. Rev. Lett. 2005, 95, 137404.

(53) Gholipour, B.; Zhang, J. F.; MacDonald, K. F.; Hewak, D. W.; Zheludev, N. I. Anall-optical, Non-volatile, Bidirectional, Phase-change Meta-switch. Adv. Mater. 2013, 25, 3050-3054.

(54) Michel, A. K.; Zalden, P.; Chigrin, D. N.; Wuttig, M.; Lindenberg, A. M.; Taubner, T. Reversible Optical Switching of Infrared Antenna Resonances with Ultrathin Phase-change Layers using Femtosecond Laser Pulses. ACS Photonics 2014, 1, 833-839.

(55) Rude, M.; Simpson, R. E.; Quidant, R.; Pruneri, V.; Renger, J. Active Control of Surface Plasmon Waveguides with a Phase Change Material. ACS Photonics 2015, 2, 669-674.

(56) Wang, Q.; Rogers, E. T. F.; Gholipour, B.; Wang, C.-M.; Yuan, G.; Teng, J.; Zheludev, N. I. Optically Reconfigurable Metasurfaces and Photonic Devices Based on Phase Change Materials. Nat. Photonics 2015, 10, 60-65.

(57) Tittl, A.; Michel, A. K. U.; Schaferling, M.; Yin, X.; Gholipour, B.; Cui, L.; Wuttig, M.; Taubner, T.; Neubrech, F.; Giessen, H. A Switchable Mid-Infrared Plasmonic Perfect Absorber with Multispectral Thermal Imaging Capability. Adv. Mater. 2015, 27, 45974603.

(58) Yin, X.; Schäferling, M.; Michel, A.; Tittl, A.; Wuttig, W.; Taubner, T.; Giessen, H. Active Chiral Plasmonics. Nano Lett. 2015, $15,4255$.

(59) Galloway, C. M.; Kreuzer, M. P.; Acimovic, S. S.; Volpe, G.; Correia, M.; Petersen, S. B.; Neves-Petersen, M. T.; Quidant, R. Plasmon-Assisted Delivery of Single Nano-Objects in an Optical Hot Spot. Nano Lett. 2013, 13, 4299-4304.

(60) Nelson, D. F. Generalizing the Poynting Vector. Phys. Rev. Lett. 1996, 76, 4713.

(61) Nye, J. F.; Berry, M. V. Dislocations in Wave Trains. Proc. R. Soc. London, Ser. A 1974, 336, 165-190.

(62) Sáenz, J. J. Optical Forces: Laser Tractor Beams. Nat. Photonics 2011, 5, 514-515.

(63) Tribelsky, M. I.; Luk'yanchuk, B. S. Anomalous Light Scattering by Small Particles. Phys. Rev. Lett. 2006, 97, 263902.

(64) Gao, D. L.; Novitsky, A.; Zhang, T.; Cheong, F. C.; Gao, L.; Lim, C. T.; Luk'yanchuk, B.; Qiu, C. W. Unveiling the Correlation between Non-diffracting Tractor Beam and its Singularity in Poynting Vector. Laser Photonics Rev. 2015, 9, 75-82.

(65) Cao, T.; Mao, L.; Gao, D.; Ding, W.; Qiu, C. W. Fano Resonant $\mathrm{Ge}_{2} \mathrm{Sb}_{2} \mathrm{Te}_{5}$ Nanoparticles Realize Switchable Lateral Optical Force. Nanoscale 2016, 8, 5657-5666.

(66) Luk'Yanchuk, B. S.; Ternovsky, V. Light Scattering by a Thin Wire with a Surface-Plasmon Resonance: Bifurcations of the Poynting Vector Field. Phys. Rev. B: Condens. Matter Mater. Phys. 2006, 73, 235432.

(67) Li, Z. P.; Zhang, S. P.; Tong, L. M.; Wang, P. J.; Dong, B.; Xu, H. X. Ultrasensitive Size-Selection of Plasmonic Nanoparticles by Fano Interference Optical Force. ACS Nano 2014, 8, 701-708.

(68) Chen, H.; Liu, S.; Zi, J.; Lin, Z. Fano Resonance-Induced Negative Optical Scattering Force on Plasmonic Nanoparticles. ACS Nano 2015, 9, 1926-1935.

(69) Xiong, F.; Liao, A. D.; Estrada, D.; Pop, E. Low-power Switching of Phase-Change Materials with Carbon Nalnotube Electrodes. Science 2011, 332, 568-570. 